\title{
Automated Detection of Alzheimer's Disease Using Wavelet Transform with Convolutional Neural Networks
}

\author{
Nur OLABI ${ }^{1 *}$, Aykut YILMAZ ${ }^{1}$, Zafer ASLAN $^{2}$
}

\begin{abstract}
Objective: Alzheimer's disease (AD) is a chronic disease that causes the death of nerve cells and tissue loss in the brain. It usually starts slowly and worsens over time. Individual computer aided systems are needed for early and accurate diagnosis of Alzheimer's. Magnetic resonance imaging (MRI) offers the opportunity to examine the pathological brain changes associated with AD. In recent years, neuroimaging data has been increasingly used to characterize $\mathrm{AD}$ with machine learning methods, offering promising tools for personalized diagnosis. Very recently, a number of studies have proposed to aid the diagnosis of AD through convolutional neural networks (CNNs).

Methods: CNN is machine learning algorithm which is used in a variety of fields, including image and pattern recognition, speech recognition, natural language processing, and video analysis. In this study Discrete wavelet transform (DWT) was used for feature extraction. DWT has attractive properties and has better image representation than other transforms like Fourier. Medical images are vulnerable to noise, they are preprocessed to remove unwanted data and improve quality. Feature extraction and classification are two essential components for the recognition system that have a significant impact on the efficiency of the system. DWT is an implementation of wavelet transform that uses a separate set of wavelet scales and translations that follow some defined rules. The aim of this study is to detect Alzheimer's disease by using convolutional neural networks and to reduce noise by preprocessing by applying DWT on the entered images.
\end{abstract}

Results: With combining DWT feature extraction and CNN algorithm for detecting Alzheimer's disease, the performance and learning rate are significantly decreased. The accuracy of the model results based on pure CNN with machine learning algorithm is higher than with than the accuracy in CNN learning without DWT future extraction. Accuracy values are $75 \%$ and $69 \%$ successively.

Conclusion: Ultimately, this study revealed that the combination of MET-PLGA NPs with current cancer therapies holds promise for the potential of breast cancer treatment.

Keywords: Alzheimer's disease, discrete wavelet transform, DWT, convolutional neural networks, CNN.

\footnotetext{
${ }^{I}$ Istanbul Aydin University, Institute of Graduate Study, Istanbul - Turkey

${ }^{2}$ Istanbul Aydin University, Faculty of Engineering, Department of Computer Engineering, Istanbul - Turkey

* Corresponding author

E-mail: nurzakiroglu@stu.aydin.edu.tr,ayilmaz@aydin.edu.tr,
}

Zafer Aslan: zaferaslan@aydin.edu.tr; ORCID:0000-0001-7707-7370,

Aykut Yılmaz:ayilmaz@aydin.edu.tr

Received: 15 November 2021 Revised: 30 November 2021 Accepted: 6 December 2021

DOI:10.17932/EJOH.2020.022/ejoh_v02i2004 


\section{Introduction}

Alzheimer's disease is a type of dementia that causes memory problems. Symptoms usually come on slowly and get worse over time, affecting a person's daily life, causing life-threatening problems.

Alzheimer's disease is a neurodegenerative disease that causes memory loss. According to the National Institute of Aging, Alzheimer's disease is defined as a progressive, irreversible brain disorder that gradually destroys memory and thinking skills, and even the ability to perform simple tasks. It usually starts in the mid-60s and is the most common cause of dementia. Factors such as age, gender, family history and heredity, education level, head trauma, Down syndrome, hypertension, depression, diabetes, alcohol, and cigarette use are stated as risk factors for Alzheimer's disease. The disease has a progressive structure and consists of various stages, and the quality of the care given is shaped according to the stage of the patient (1).

With aging, forgetfulness, slowing of speech, weakness, unhappiness, and increased sleepiness can be seen in people. However, these symptoms may be signs of $\mathrm{AD}$, and $\mathrm{AD}$ is not a temporary disease. It progresses in a variable way from person to person; It makes the life of patients very difficult and seriously reduces the quality of life (2).

The most important feature of AD is that it starts without specific symptoms and progresses slowly.

Patients and their relatives cannot tell the exact time of onset of the complaints. With the thought that forgetfulness is normal in old age, it becomes difficult to detect the onset of $\mathrm{AD}$. Therefore, the time to consult a physician is also delayed (3).

In the health sector, it is not possible to manage diagnosis and diagnosis only with human power. Machine learning techniques, one of the artificial intelligence technologies, are widely used in medical predictions (4). With machine learning techniques, rapid and reliable disease prediction can be made.

There are many algorithms of machine learning, and it is decided which algorithm to use according to the source of the problem and the amount of data. Different algorithms can produce specificity and sensitivity values according to the data used (5). Considering these values according to the data used, the best fit among the different algorithms is selected. In this way, the best algorithm is preferred, and the result is improved, and the time cost is reduced (5). 
Wavelet transform is a type of transform used for time-frequency analysis of a signal. Especially the problems experienced during the examination of seismic data allowed the development of the "Continuous Wavelet Transform". Later, wavelet analysis was found to be applicable to a wide variety of signal types (6). In the studies conducted, wavelet transform has been used in the diagnosis of discontinuity in signals (interpretation of heart graphs can be given as an example) and in noise removal from the signal (7).

Discrete Wavelet Transform DWT can be considered as the best transform field technique among other techniques due to its multi-resolution properties and excellent time-frequency analysis and is widely used for signal processing purposes (8), (9). When an image is transformed by wavelet, 4 sub bands (low frequency component LL, horizontal detail component HL, vertical detail component $\mathrm{LH}$, and diagonal detail component $\mathrm{HH}$ ) are obtained. Because most of the image energy is concentrated in the low-frequency sub band, embedding the stamp in the low-frequency sub band causes perceptual changes in the image. However, if the stamp is embedded in the high frequency sub bands instead of the low frequency band, the durability of the stamping scheme is adversely affected. For this reason, the LL sub band, which is more resistant to many attacks than other sub bands, was preferred in this study.

The Discrete Wavelet Transform (DWT) of image signals produces a nonredundant image representation, which provides better spatial and spectral localization of image formation, compared with other multi scale representations such as Gaussian and Laplacian pyramid. Recently, Discrete Wavelet Transform has attracted more and more interest in image de-noising (10).

Similarly to this study there are several studies that applied to detect the AD disease using machine learning techniques, like the study done by Jha, Kim, Kwon (11) for diagnosis AD disease using wavelet transform, they used DualTree Complex wavelet transform for image processing, the results showed that the proposed method achieved an accuracy of $90.06 \pm 0.01 \%$, a sensitivity of $92.00 \pm 0.04 \%$, a specificity of $87.78 \pm 0.04 \%$, and a precision of $89.6 \pm 0.03 \%$ and outperformed 7 state-of-the-art algorithms.

\section{Data and Methodology}

The aim of this study is to detect Alzheimer's disease using image processing and $\mathrm{CNN}$ machine learning. In the $\mathrm{CNN}$ algorithm, preprocessing will be done on the image using the wavelet's DWT library. DWT will be used to remove the noises. 


\section{Dataset}

The dataset is taken from the Kaggle website. The data set, consisting of 6400 brain tomography scans, consists of normal people, people with moderate Alzheimer's disease, and people with severe Alzheimer's disease (12). In this study, we divided the brain tomography of a person into four classes as

i) normal tomography (have no Alzheimer‘s disease),

ii) mild disease,

iii) moderate disease,

iv) high-grade disease.

Table 1 shows descriptive statistics of images that used in testing operation of the learning of model.

Table 1. Descriptive statistics of testing images

\begin{tabular}{clcc}
\hline Class Number & Class Description & Images Count & Used Images (\%) \\
\hline 1 & Normal Tomography & 640 & $\% 50$ \\
\hline 2 & Mild Disease & 179 & $\% 14$ \\
\hline 3 & Moderate Disease & 12 & $\% 1$ \\
\hline 4 & High-grade Disease & 448 & $\% 35$ \\
\hline Total & & 1279 & $\% 20$ \\
\hline
\end{tabular}

Table 2. Descriptive statistics of training images

\begin{tabular}{clcc}
\hline Class Number & Class Description & Images Count & Used Images (\%) \\
\hline 1 & Normal Tomography & 2560 & $\% 50$ \\
\hline 2 & Mild Disease & 717 & $\% 14$ \\
\hline 3 & Moderate Disease & 52 & $\% 1$ \\
\hline 4 & High-grade Disease & 1792 & $\% 35$ \\
\hline Total & & 5121 & $\% 80$ \\
\hline
\end{tabular}


Figure 1 shows sample of normal brain and High-grade of AD disease brain tomography that used in our study, The brain affected by Alzheimer's is considerably shrunken, due to the degeneration and death of nerve cells.

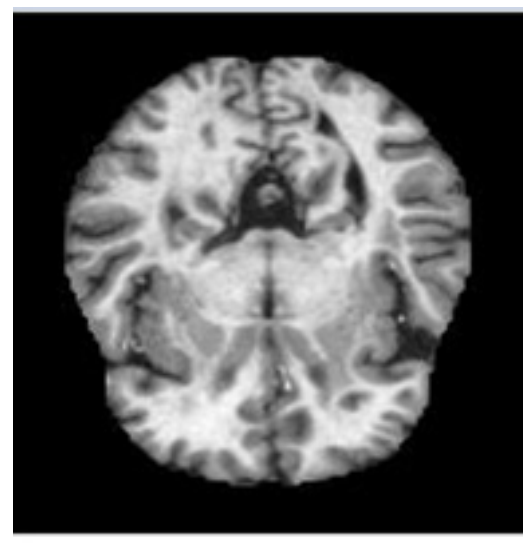

Normal Brain

Tomography

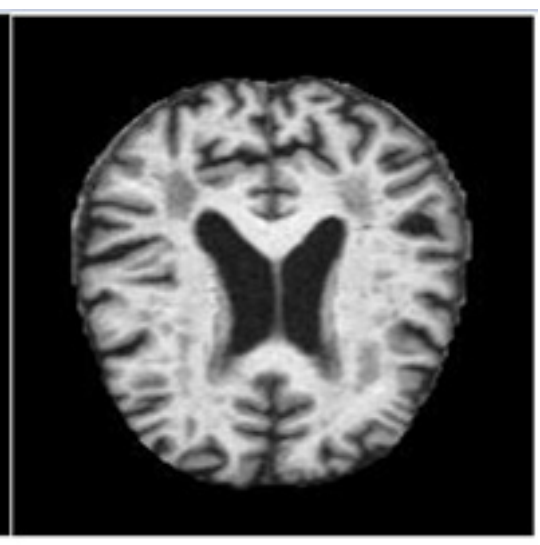

AD Disease Brain Tomography

Figure 1. Normal brain vs. Alzheimer's Disease

\section{Image Processing}

Image processing is a method of converting an image into digital form and performing operations on it to obtain an enhanced image or extract some useful information from the image. It is a type where the input is an image, some kind of signal distribution, such as a video frame or a photograph, and the output can be the image, or the characteristics associated with that image. Digital x-ray is used to capture images, scan the image, or store the image in digital format like jpeg, png, so it is useful in diagnosing thepatients' disease. However, digital $\mathrm{x}$-ray images contain images such as gaussian noise or salt and pepper noise, so they sometimes do not give a clear picture of the $\mathrm{x}$-ray image. Therefore, image processing is important to remove noise in the image to improve the quality of images (13). 


\section{Convolutional Neural Networks (CNN)}

Artificial Neural Networks (ANNs) are computational systems heavily inspired by the work of biological nervous systems (like the human brain). ANNs essentially consist of a large number of interconnected computational nodes (called neurons) where jobs are distributed in a distributed manner to learn collectively from the inputs in order to optimize the final output. The basic structure of an ANN can be modeled as shown in Figure 2. It is loaded in such a way as to distribute the input layer to the hidden layers, usually in the form of a multidimensional vector. The hidden layers then make decisions from the previous layer and discuss how a stochastic change within them harms or improves the final output, this is called the learning process (14).

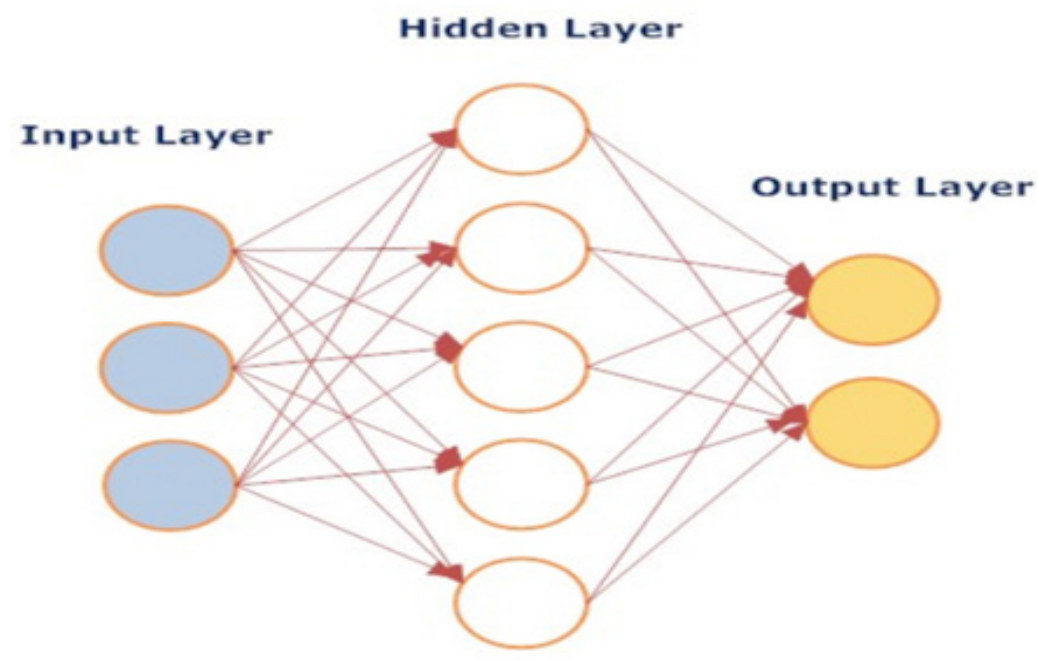

Figure 2. ANN basic structure

Convolutional Neural Networks (CNNs) are like traditional ANNs in that they consist of neurons that optimize themselves through learning. Each neuron will again receive an input and perform an operation that is the basis of countless ANNs. The last layer contains the loss functions associated with the classes. The only significant difference between CNNs and ANNs is that CNNs are primarily used for pattern recognition in images. This allows us to encode imagespecific features into the architecture, making the network more suitable for image-oriented tasks, while further reducing the parameters required to tune the 
model. One of the major limitations of traditional ANN forms is that they tend to struggle with the computational complexity required to compute image data (15). Convolutional Neural Network has produced groundbreaking results in various fields related to pattern recognition in the last decade. The most useful aspect of CNNs is to reduce the number of parameters in ANN. This success has led both researchers and developers to approach larger models to solve complex tasks which are not possible with classical ANNs. The most important assumption regarding problems solved by $\mathrm{CNN}$ should not have features that are spatially dependent. In other words, in a face recognition app, for example, we don't need to pay attention to where the faces are in the pictures. The only concern is to detect them regardless of their position in the given images. Another important aspect of CNN is to obtain abstract features when the input spreads to deeper layers (16). CNNs mainly focus on comparing input with images. It focuses on finding the architecture that will best fit the need to deal with specific data types. One of the most important differences with ANN is that the layers within the $\mathrm{CNN}$ consist of neurons arranged in three dimensions; The spatial dimensions of the inputs are height, width, and depth. Depth corresponds to the third dimension of an activation volume, not the total number of layers in the ANN. Neurons in any layer connect only to a small region of the layer before it.

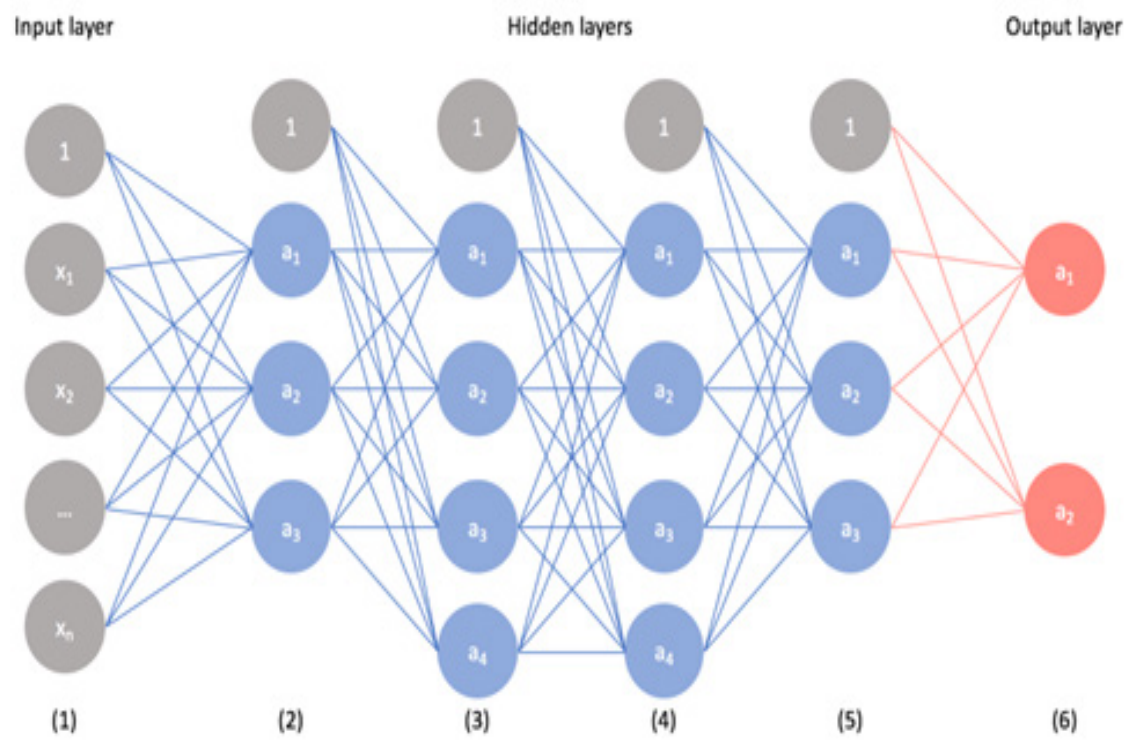

Figure 3. CNN basic structure 


\section{Results and Discussion}

Medical images are vulnerable to noise. Therefore, noise suppression is considered a challenging task in the medical field. Medical images are preprocessed to remove unwanted data and improve quality. Feature extraction and classification are two essential components for the recognition system that have a significant impact on the efficiency of the system. In this study, DWT was used to extract relevant features. Figure 4 shows the block diagram of the proposed AH detection method. The proposed method includes the following processes: Feature extraction Feature reduction and Classification.

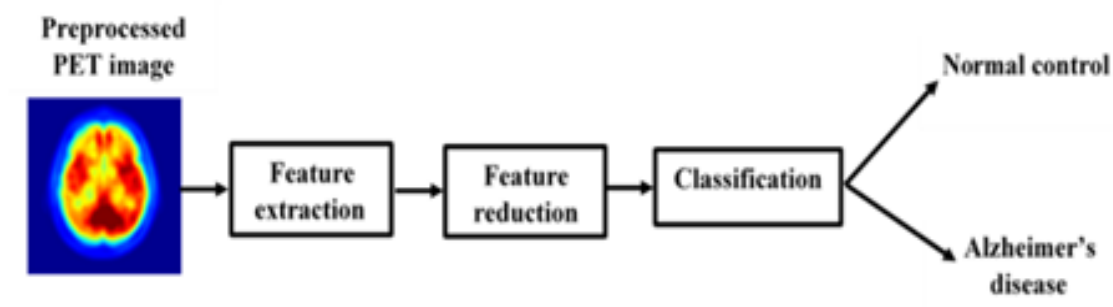

Figure 4. Block diagram of the proposed method (15)

\section{Feature extraction}

Feature extraction is designed to extract compact and meaningful information from the image and reduce the size by eliminating noise and unwanted data. DWT is a powerful mathematical tool that gives a multi-resolution representation of any signal or image. It decomposes the signal or image into a different frequency component and then probes each component with a resolution appropriate to its scale (17). DWT is derived from continuous wavelet transform (CWT), suitable for analysis of medical image. The strength of DWT is the use of separate sets of scales and shifts that provide sufficient information and reduce the computational load (19). In figure 5, DWT library was applied on one sample of brain MRI.
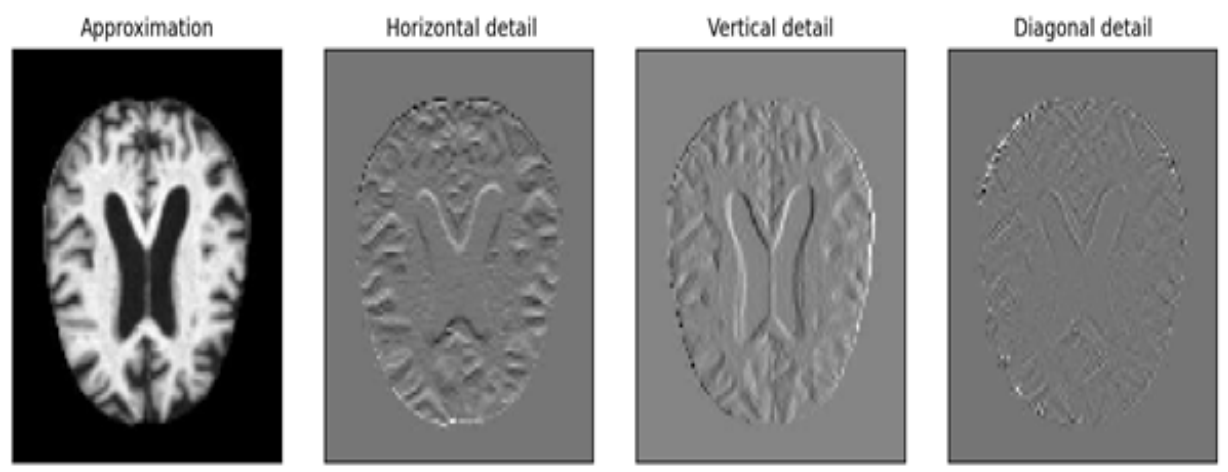

Figure 5. Sample image preprocessing 


\section{Conclusion and Suggestions}

$\mathrm{CNN}$ algorithm was used to detect $\mathrm{AD}$ disease with machine learning, the model was processed with 6400 brain tomography, 4098 images were used for training set, 1023 images for validation and 1279 images for testing. The accuracy rate was calculated as $75 \%$. In Figure 6, there are results showing the accuracy. The AUC (Area Under Curve) diagram was also calculated. The most popular and simple method used to measure model performance is the accuracy rate of the model. It is the ratio of the number of correctly classified samples (TP $+\mathrm{TN})$ to the total number of samples $(\mathrm{TP}+\mathrm{TN}+\mathrm{FP}+\mathrm{FN})$. In other words, it is the ratio of the number of misclassified samples $(\mathrm{FP}+\mathrm{FN})$ to the total number of samples $(\mathrm{TP}+\mathrm{TN}+\mathrm{FP}+\mathrm{FN})(17)$.

$$
\begin{aligned}
& \text { Error Rate }=\frac{F P+F N}{T P+F P+F N+T N} \\
& \text { Accuracy }=\frac{T P+T N}{T P+F P+F N+T N}
\end{aligned}
$$
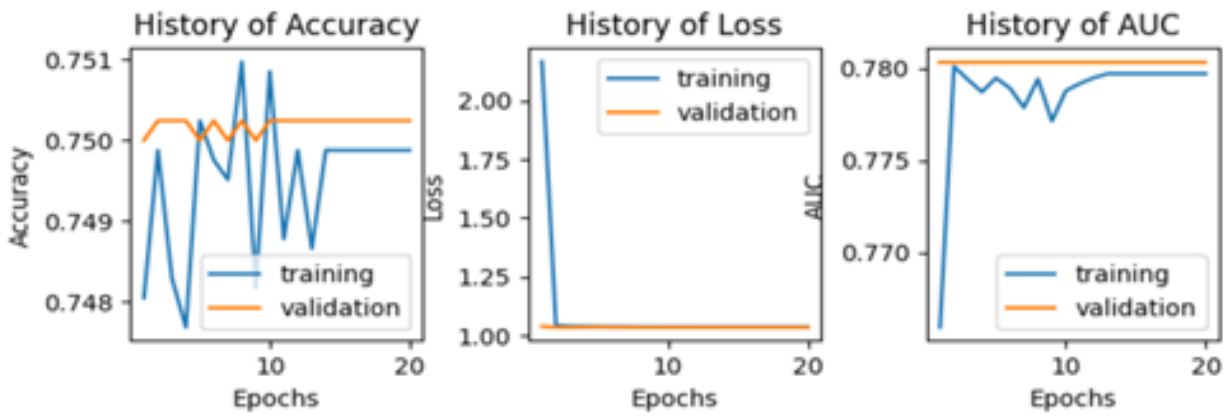

Figure 6. Model training results

We also used sensitivity and specificity measures to evaluate the performance of the classifiers.

Sensitivity $=\mathrm{TP} /(\mathrm{TP}+\mathrm{FN})=($ Number of true positive assessment $) /($ Number of all positive assessment).

Specificity $=\mathrm{TN} /(\mathrm{TN}+\mathrm{FP})=($ Number of true negative assessment $) /($ Number of all negative assessment). 
Automated Detection of Alzheimer's Disease Using Wavelet Transform with Convolutional Neural Networks

Table 3. Comparing results between Pure CNN and CNN with DWT

\begin{tabular}{cccc}
\hline Method & Accuracy & Sensitivity & Specificity \\
\hline Pure CNN & 0.75 & 0.50 & 0.84 \\
\hline CNN with DWT & 0.69 & 0.53 & 0.80 \\
\hline
\end{tabular}

It was determined that the learning time was 4 hours longer than the CNN algorithm was used with the Wavelet in Dwt library. The DWT library is used instead of MaxPooling, which is the last layer of CNN. The development has been done with Python language and the accuracy rate has reached up to $69 \%$. Thus, we found that the normal $\mathrm{CNN}$ algorithm provides better performance when compared to the use of pure CNN and DWT with CNN. We can select and test different wavelet libraries to increase the accuracy.

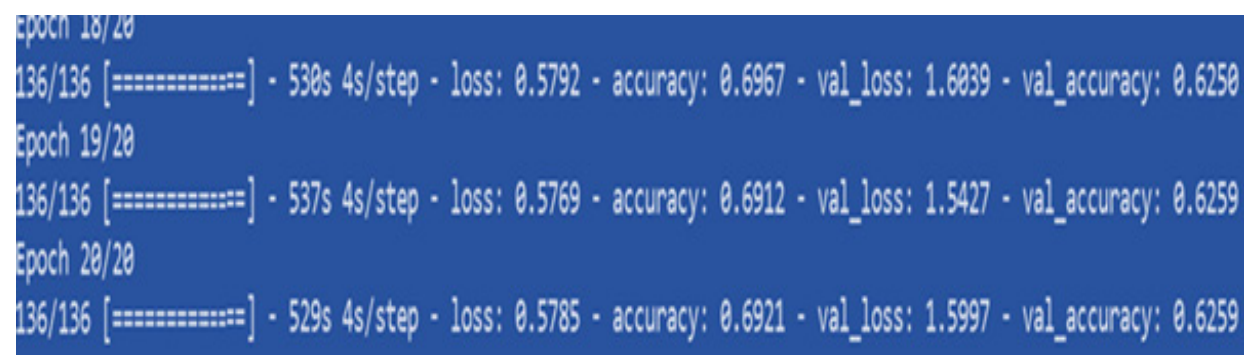

Figure 7. Sampling of output data based on training result.

For future work we can use different machine learning algorithms to compare the results and get a better accuracy by using different libraries of the wavelet.

\section{Acknowledgement}

This paper was presented at IWW2021 International Conference, 23 -24 June 2021, IAU.

\section{References}

1. Soner S, Aykut S. Alzheimer Hastalık Sürecinde Bakım Veren Aile Üyelerinin Yaşadıkları Güçlükler ve Sosyal Hizmet, Ahi Evran Üniversitesi Sosyal Bilimler Enstitüsü Dergisi (AEÜSBED). 2017;3(2):375-387.

2. Wang J, Gu BJ, Masters CL, Wang YJ. A systemic view of Alzheimer disease insights from amyloid- $\beta$ metabolism beyond the brain. Nature Reviews Neurology. 2017;13:612-623.

3. Şahin HA. Alzheimer hastalığını klinik belirtileri ve seyri. Türkiye Klinikleri. 2009;2(1):31- 35 . 
4. Islam T, Raihan M, Farzana F, et al. An Empirical Study on es Mellitus Prediction for Typical and Non-Typical Cases using Machine Learning Approaches. 10th ICCCNT 2019.

5. Chubak J, Pocobelli G, Weiss NS. Trade-offs between accuracy measures for electronic healthcare data algorithms. J Clin Epidemiol. 2012;65(3):343-349.

6. Yong-xiang Z, Xiao-xu Z, Hui-mei Y, Wei-gong Z. Wavelet Packet Threshold Approach to Denoising Piezoelectricity Gyro Signal. International Conference on Computer Engineering and Technology ICCET 09, Singapore. 2009: pp. 266 $-269$.

7. Taswell C. The What, How, and Why of Wavelet Shrinkage Denoising. Technical Report 1999;CT-1998-09.

8. Cai Y, Guo W, Ding H. Dwt-svd. Journal of Software. 2013;8(7):1801.

9. Debnath L. Brief historical introduction to wavelet transforms. International Journal of Mathematical Education in Science and Technology. 1998;29(5);677688.

10. Available at: https://www.kaggle.com/tourist55/alzheimers-dataset-4-classof images. Accessed 09.10.2021.

11. Chikhalekar AT. Analysis of Image Processing for Digital X-Ray. International Research Journal of Engineering and Technology (IRJET). 2016;3(5):1364-1368.

12. Gunawardena N, Kodikara N, Rajapakse R. Applying convolutional neural networks for pre-detection of alzheimer's disease from structural MRI data. 24th International Conference on Mechatronics and Machine Vision in Practice. 2017.

13. O'Shea K, Nash R. An Introduction to Convolutional Neural Networks Available at: https://arxiv.org/abs/1511.08458. Accessed 01.11.2021.

14. Albawi S, Mohammed TA, Al-Zawi S. Understanding of a convolutional neural network. 2017 International Conference on Engineering and Technology (ICET), 2017, pp. 1-6

15. Akbar S, Rajeswari R. Diagnosis of Alzheimer's Disease Using Wavelet Transform and Artificial Neural Network. Journal of Advanced Research in Dynamical and Control Systems. 2019;11(7):7.

16. Aggarwal N, Agrawal RK. First and Second Order Statistics Features for Classification of Magnetic Resonance Brain Images. Journal of Signal and Information Processing. 2012;3:146-153.

17. Coşkun C, Baykal A. Veri Madenciliğinde Sınıflandırma Algoritmalarının Bir Örnek Üzerinde Karşılaştırılması. XIII. Akademik Bilişim Konferansı Bildirileri. 2011. 